Revue

Revue de l'histoire des religions

de Ihistoire des religions

$2 \mid 2009$

Varia

\title{
Dominique Urvoy, Histoire de la pensée arabe et
} islamique

Paris, Le Seuil, 2006, 676 p., 22 cm, $29 €$.

Geneviève Gobillot

\section{CpenEdition}

Journals

Édition électronique

URL : http://journals.openedition.org/rhr/7243

DOI : $10.4000 /$ rhr.7243

ISSN : 2105-2573

Éditeur

Armand Colin

Édition imprimée

Date de publication : 1 avril 2009

Pagination : 270-273

ISBN : 978-2200-92590-1

ISSN : 0035-1423

Référence électronique

Geneviève Gobillot, «Dominique Urvoy, Histoire de la pensée arabe et islamique », Revue de l'histoire des religions [En ligne], 2 | 2009, mis en ligne le 12 janvier 2010, consulté le 22 septembre 2020. URL: http://journals.openedition.org/rhr/7243; DOl : https://doi.org/10.4000/rhr.7243 
tien avec l'Archimandrite Job Gechta, professeur à l'Institut Saint-Serge à Paris. Une bibliographie et un index onomastique complètent cet ouvrage utile.

Stéphane-Marie MorGaIN, Institut catholique de Toulouse.

Dominique Urvor, Histoire de la pensée arabe et islamique, Paris, Le Seuil, 2006, 676 p., $22 \mathrm{~cm}, 29 €$.

L'Histoire de la pensée arabe et islamique est avant tout une grande synthèse «classique » destinée à traverser le temps, à l'instar de Les Schismes dans l'islam d'Henri Laoust, qu'elle complète et prolonge pour les questions essentielles.

Son auteur, qu'il n'est plus nécessaire de présenter aux arabisants, peut néanmoins l'être utilement aux autres spécialistes auxquels il destine cet ouvrage, comme il l'indique dans sa préface. Dominique Urvoy est une figure particulièrement reconnue et respectée, non seulement au sein de l'élite restreinte des islamologues contemporains, mais aussi dans les cercles internationaux des philosophes et des historiens pour tout ce qui touche au domaine de l'histoire des idées. Philosophe de formation et actuellement professeur à l'Université de Toulouse le Mirail, il s'est penché sur un éventail de questions très large, allant de la période de formation des textes fondateurs de l'islam aux réflexions actuelles sur les problèmes de la bioéthique ou sur le dialogue islamo-chrétien par exemple, toutes problématiques exigeant une culture d'une ampleur considérable. Il était donc l'un des rares spécialistes contemporains à réunir toutes les aptitudes requises pour la réalisation d'un tel ouvrage, et ce d'autant plus qu'il s'est déjà essayé à un certain nombre de synthèses qui l'ont fait connaître d'un public beaucoup plus large que celui des cercles académiques où il fait autorité. On citera, par exemple : Les penseurs libres dans l'islam classique (1996); Averroes : les ambitions d'un intellectuel musulman (1998); Les mots de l'islam (2004).

Ainsi qu'il l'explique lui-même, sa démarche se distingue de toutes celles qui ont été adoptées à ce jour dans la mesure où il s'agit d'un « essai de synthèse des perspectives intellectuelles » qui, loin de se cantonner à la description des doctrines successives, propose de « saisir le mouvement collectif qui passe à travers chacune en la confrontant à des formes de pensée comparables ou, au contraire, adverses ».

C'est en fait un paysage complet que brosse ici l'auteur, avec ses reliefs, ses perspectives, ses jeux d'ombre et de lumière et ses lignes de fuite, mais aussi stylisé, ordonné et organisé autour d'une réalité essentielle, à savoir : « la force prise, dans la pensée arabo-musulmane, par toute idée déjà énoncée ", qui la rend susceptible de resurgir à n'importe quel moment de l'histoire si le contexte s'y prête. C'est ainsi que figurent à un premier plan de dimensions importantes (presque les deux tiers de l'ouvrage), les sources et les racines, correspondant aux périodes les plus 
anciennes de formation des idées et des problématiques fondamentales de la pensée arabo-islamique jusqu'au quatrième siècle de l'Hégire. Au second plan figurent les mouvements de pensée principaux et les personnalités les plus marquantes qui ont contribué au développement de ces idées et en ont, jusqu'au-dix huitième siècle (XII ${ }^{\mathrm{e}}$ siècle de l'Hégire), éventuellement apporté de nouvelles. Ces huit siècles occupent presque entièrement le dernier tiers de l'ouvrage. Enfin, en troisième plan, un peu moins d'une centaine de pages sont consacrées à la pensée arabo-musulmane depuis les mouvements réformistes du XIX ${ }^{\mathrm{e}}$ siècle.

Cette manière de procéder est sans aucun doute la mieux adaptée au sujet, car elle permet de donner la vision la plus juste et la plus scientifique de son objet tout en limitant au maximum les répétitions qui sont en effet l'une des caractéristiques les plus marquantes de la pensée araboislamique. D'une part, elle permet de mettre clairement en évidence toutes les combinaisons, synchroniques ou diachroniques, qui relient les idées entre elles, mais également les idées et les destinées de certains groupes ou individus, ou encore le contexte politico-social avec les personnes et les modes de pensée. On peut en donner pour exemple (p. 463) la correspondance mise en évidence, chez Ibn Tufayl, entre options philosophiques et positions politiques de circonstance. Aucun élément n'est négligé, ni occulté. Simplement, une attention particulière est apportée aux reliefs les plus saillants de cette évolution chronologique de la pensée, qui dessinent d'eux-mêmes les grandes lignes du panorama envisagé par l'auteur.

L'une des particularités les plus remarquables de l'ouvrage est la juste place faite aux penseurs juifs et chrétiens, intégrés de plein droit à cet ensemble de par leur expression en langue arabe. Quelques grandes figures : Jean Damascène, Yahyâ Ibn Adî, $\mathrm{Sa}^{\mathrm{c}}$ adiyya Gaon, Maïmonide, Ibn Kammûna, émergent, jetant une lumière nouvelle sur des aspects de la pensée arabo-musulmane jusque là ignorés ou négligés par de nombreux spécialistes : les polémiques, les influences réciproques, les coïncidences et les divergences. Tous les outils sont donnés pour comprendre la teneur des contacts intellectuels et sociaux entre musulmans et non-musulmans (tributaires) aux périodes envisagées.

Cette même précision est bien entendu présente à tous niveaux. Une fois les idées principales énoncées et explicitées, il est possible de suivre leur devenir tout au long de l'ouvrage. Il s'agit tout d'abord des problématiques qui n'ont jamais cessé d'être d'actualité comme les questions relatives au libre arbitre et à la prédestination, à la fonction de calife et aux relations entre pouvoir et religion ou les problèmes liés à l'idée d'orthodoxie. On peut citer ensuite les démarches novatrices en leur temps, mais totalement abandonnées pour des raisons diverses, le plus souvent parce qu'elles ne pouvaient être conciliées avec les exigences du milieu religieux dominant, comme les prises de position d'Ibn Sab'în vis-à-vis du christianisme en particulier. Enfin, les systèmes de pensée ou les doctrines qui, ignorées au moment de leur élaboration, ont ressurgi des décennies, quelques fois des siècles, plus tard, au gré des circonstances, comme le concept de « Sceau de la sainteté » d'al-Hakîm al-Tirmidhî, sorti de l'oubli par Ibn ' Arabî. 
Dominique Urvoy ne se contente pas de proposer une « histoire de la pensée de l'élite ». A plusieurs reprises il pose le problème de la réception des idées philosophiques, théologiques ou mystiques par les masses, donc de l'histoire des mentalités, bien qu'il excelle tout particulièrement dans la présentation des penseurs libres et dans la mise en évidence des traits saillants des systèmes les plus originaux. Certains chapitres, comme par exemple le IX, consacré à l'assimilation du savoir étranger, la présentation analytique du mouvement $\mathrm{mu}^{\mathrm{c}}$ tazilite à ses débuts et la formation de la théologie juive en regard du kalâm, ou encore la réflexion épistémologique du chapitre XVIII sont des modèles du genre. Irremplaçables propédeutiques à la réflexion personnelle et à l'approfondissement des questions, ils recèlent des qualités pédagogiques inestimables qui confirment le statut de l'ouvrage comme point de départ indispensable de toute étude, universitaire ou non, de la civilisation arabo-musulmane. Néanmoins, en raison d'autres qualités méthodologiques, en particulier dans la mesure où il indique très nettement à la fois toutes les ouvertures possibles et les limites de la grande majorité des questions abordées, il s'impose comme un outil indispensable aux chercheurs des niveaux les plus avancés.

Pourtant, l'ouvrage est écrit avec clarté, simplicité et concision et recèle de nombreux raccourcis riches d'enseignement. Il demande seulement à être lu avec une attention soutenue, qui rappelle que le savoir digne de ce nom ne peut jamais être «bradé ». En effet, en dépit de son caractère tout à fait « classique », de par la place conférée aux principaux penseurs musulmans proportionnellement à leur représentativité d'ensemble : alKindî, al-Hakîm al-Tirmidhî, al-Fârâbî, Avicenne, Averroès, al-Ghazâlî, Ibn Hazm, Ibn Taymiyya, Ibn Sabcîn, Ibn Khaldûn, Mollâ Sâdra Shirâzî, Nasir Hâmid Abû Zayd, Dominique Urvoy a introduit au fur et à mesure de ses développements, les résultats des découvertes et des théories les plus récentes sur chaque question. Par cette manière de procéder il réalise, page après page, une véritable remise à jour des connaissances et relègue définitivement un grand nombre d'idées reçues, qui malheureusement encombrent encore de nombreux travaux sur l'histoire des idées arabomusulmanes.

On notera quelques points particulièrement dignes de retenir l'attention.

Tout d'abord le constat que ce sont les chrétiens qui ont inventé l'écriture spécifique arabe en vue de « rédiger des florilèges bibliques et des recueils liturgiques qui ont marqué les scribes, chargés à l'époque omeyyade, de composer le recueil coranique » (p. 196). L'auteur renvoie sur ce point aux dernières publications du regretté Louis de Prémare (en particulier Les fondations de l'islam, Paris, 2002).

Il faut souligner ensuite l'idée, tout à fait essentielle pour le développement ultérieur de la théologie musulmane, que, pour résoudre le problème métaphysique de l'irruption de la transcendance dans l'immanence, «l'islam se rapproche du christianisme, en envisageant « la 'descente' d'un Livre qui est bien une forme d'incarnation divine » (p. 49).

De même, il n'était pas inutile de rappeler, comme le fait Dominique Urvoy, le caractère relativement tardif de la formation de l'idée de 
« sunnisme » (p. 330), processus connu depuis longtemps, mais encore souvent occulté par les historiens de la pensée au profit de la conception vague, mais fortement ancrée dans les mentalités, d'un «sunnisme originel » allant de pair avec l'ancienneté de l'ensemble des traditions prophétiques. Dominique Urvoy replace cette situation dans son contexte véritable en remarquant (p. 319) que : « l'esprit sémitique qui a présidé à la fabrication d'une multitude de hadîth prophétiques » consiste dans l'idée que « l'islam doit contenir tout ce qui est jugé bon ». Cette tendance au « rassemblement des données » se retrouve, comme il le souligne un peu plus loin, chez plusieurs penseurs musulmans, comme Ghazâlî ou, sous une autre forme, chez Ibn ${ }^{\mathrm{c}}$ Arabî, dont il résume ainsi la pensée : « son projet est dans l'aménagement d'un univers mental préexistant pour obtenir un monde cohérent de part en part, sans point de départ, ni point d'arrivée » (p. 518).

Les exemples de ce genre, qui constituent en réalité les structures profondes du texte, sont trop nombreux pour être tous évoqués ici. On conclura donc en évoquant le problème philosophique qui parcourt l'ouvrage du début à la fin et que l'auteur propose comme dernier sujet de méditation, dans ses toutes dernières lignes, à savoir que : « la pensée arabe, quelle que soit la foi de celui qui s'exprime, et celle des musulmans non arabophones » a toujours un caractère affirmatif et qu'en toute situation elle est dirigée vers le dehors car « par elle-même, elle ne connaît pas le tragique » (p. 655). Cette réflexion fait, en quelque sorte, rebondir in extremis le débat autour de la langue arabe et de ses rapports avec l'islam posés au début du livre. Si l'auteur ne donne pas directement les clés permettant d'accéder à tout ce que recouvre, pour lui, cette absence de tragique, dans l'attente de ses publications à venir on peut déjà trouver des indices relatifs à ses causes dans les passages qu'il consacre à la réflexion sur la langue au chapitre IX, à savoir que d'une part, l'arabe, en tant que langue sémitique fait « surgir le sens de l'intérieur même des mots, exprimant par le jeu de la flexion interne ce que l'indo-européen développe en une périphrase » (p. 155). D'autre part, il constate que, jusqu'à la période des grandes traductions des textes philosophiques et scientifiques grecs, «l'histoire de cette langue l'avait consacrée à la poésie et à l'éloquence, c'est-à-dire à l'image et à la sonorité », ensemble de facteurs qui pourrait expliquer sa tendance à exprimer une pensée de « l'extériorité », précisément au moment où furent rédigés les textes fondateurs de l'islam, lesquels sont restés l'expression religieuse fondamentale de référence pour tous les arabophones, qu'ils soient musulmans ou non et, bien entendu, pour tous les musulmans, même ignorants de l'arabe. On découvre donc avec surprise, mais non sans enthousiasme, que les perspectives de réflexions à venir ouvertes par ce livre sont au moins aussi vastes que le savoir et l'intellection subtile des phénomènes qu'il vient de proposer à son lecteur attentif.

Geneviève GobILlot, Université de Lyon. 